\title{
L-dopa and carbidopa (sinemet) in the management of parkinsonism
}

\author{
EDMUND CRITCHLEY \\ D.M., M.R.C.P.
}

\author{
Department of Neurology, Royal Infirmary, Preston
}

\begin{abstract}
Summary
Parkinsonism is a degenerative disease. A weakening in the responsiveness to dopamine replacement therapy affects many patients: thus $\mathbf{4 5} \%$ fail to maintain their initial improvement after 2 years' and $50 \%$ after 3 years' medication. However, by reducing the peripheral and systemic side actions of dopamine through the selective introduction of sinemet, a decarboxylase inhibitor + L-dopa combination, a partial amelioration of as much as $35 \%$ at 2 years, or $20 \%$ at 3 years, can be achieved. There is evidence, also, that the initial choice of anti-parkinsonian therapy may be critical to the long term prognosis.
\end{abstract}

\section{Introduction}

The morbidity of parkinsonism is only in part dependent upon a failure of dopamine synthesis. Although replacement therapy with L-dopa will result in an alleviation of symptoms until the terminal stages of the disease, the initial improvement is gradually replaced by a period wherein the parkinsonian disabilities continue to increase despite the retention of partial responsiveness to sustained medication (Barbeau, 1969; Hunter et al., 1973).

Furthermore, the administration of L-dopa may be attended by undesirable central and peripheral effects which are dose related and depend upon the metabolism of L-dopa away from the striatal target cells. Sinemet-a combination of L-dopa with carbidopa, a decarboxylase inhibitor-provides a more rapid response at a lower total dosage of the active principle, thereby diminishing the likelihood of systemic side actions. Marsden, Parkes and Rees (1973), as a result of an extended study of L-dopa combined with carbidopa compared with L-dopa alone, concluded that the combined treatment is the therapy of choice for patients who require L-dopa. The present report concerns the long term management of patients on L-dopa with special reference to the introduction of combined L-dopa and carbidopa therapy in selected cases.

\section{Patients}

When sinemet became generally available, 111 patients (fifty-three men and fifty-eight women, average age 65.4 years) currently attending the out- patient department were re-examined to see whether they might benefit from the introduction of sinemet. A change of medication was suggested where there had been a decline in the effectiveness of L-dopa, an inadequate initial response, gastro-intestinal or postural side effects, a poor tolerance of the drug, or because the treatment involved too many tablets, perhaps leaving a taste in the mouth or staining clothing. In the case of those experiencing 'on-off' effects, dyskinesias or in whom mental symptoms were beginning to appear, a change of medication was approached more tentatively by the physician but often sought by the patient or relatives. Where the patient was clearly satisfied with the treatment or where it had been decided to circumvent the dyskinetic or other side effects by careful adjustment of the timing and dosage of the medication, na change in therapy was contemplated.

At the conclusion of the discussion between patient and physician, thirty-eight patients continued with L-dopa, seventy-three explored the effect of sinemet, and twenty-four new patients, who had not previously received L-dopa, began treatment with sinemet.

The majority of the 135 patients, whether on Ldopa or sinemet, were also receiving anticholinergic drugs; many of those who had not responded satisfactorily to L-dopa had received amantadine; and, irrespective of the measure of control of the physical symptoms of parkinsonism, thirty patients had received or were receiving tricyclic antidepressants.

\section{Response to L-dopa}

The response to therapy was studied using a clinical rating developed from a quantitative assessment of tremor, rigidity, hypokinesia, mood and speech and balancing improvement against side effects (Critchley, 1972).

All 111 patients on L-dopa were assessed after 9 months and again at 2 years. Initially, $49.4 \%$ showed a definite (excellent or good) and $27.2 \%$ a moderate improvement. By 2 years the proportions had fallen to $19.2 \%$ and $25.3 \%$, respectively. Forty-two patients were reassessed after 3 years' therapy. Their initial level of response had been $57 \%$ definitely improved and $29 \%$ moderately so. At 3 years, only 
TABLE 1. Patients initially treated by L-dopa alone. (a) One hundred and eleven patients: duration of treatment on L-dopa alone, 2 years; (b) forty-two patients: results in these treated for 3 years with L-dopa alone; (c) nineteen patients: duration of parkinsonism, 10 years; average time on L-dopa alone, 2 years

\begin{tabular}{|c|c|c|c|c|c|c|}
\hline \multirow[b]{2}{*}{ Improvement } & \multicolumn{2}{|c|}{ Early } & \multicolumn{2}{|c|}{ Late } & \multicolumn{2}{|c|}{ Addition of sinemet } \\
\hline & No. & $\%$ & No. & $\%$ & No. & $\%$ \\
\hline (a) & & & & & \multicolumn{2}{|c|}{ (73 pts) } \\
\hline Excellent & 23 & 21 & 7 & 6 & 14 & 13 \\
\hline Good & 32 & 29 & 15 & 14 & 28 & 25 \\
\hline Moderate & 30 & 27 & 28 & 25 & 37 & 33 \\
\hline Slight & 19 & 17 & 46 & 41 & 21 & 19 \\
\hline None & 7 & 6 & 15 & 14 & 11 & 10 \\
\hline Total & 111 & & 111 & & 111 & \\
\hline (b) & & & & & \multicolumn{2}{|c|}{ (29 pts) } \\
\hline Excellent & 12 & 29 & 1 & 2 & 2 & 5 \\
\hline Good & 12 & 29 & 4 & 9 & 9 & 21 \\
\hline Moderate & 12 & 29 & 13 & 31 & 14 & 33 \\
\hline Slight & 3 & $6 \cdot 5$ & 17 & 40 & 10 & 23 \\
\hline None & 3 & $6 \cdot 5$ & 7 & 18 & 7 & 18 \\
\hline Total & 42 & & 42 & & 42 & \\
\hline (c) & & & & & \multicolumn{2}{|c|}{ (15 pts) } \\
\hline Excellent & 1 & 5 & 0 & 0 & 0 & 0 \\
\hline Good & 8 & 42 & 3 & 16 & 5 & 26 \\
\hline Moderate & 6 & 32 & 4 & 21 & 7 & 37 \\
\hline Slight & 3 & 16 & 10 & 52 & 5 & 26 \\
\hline None & 1 & 5 & 2 & 11 & 2 & 11 \\
\hline Total & 19 & & 19 & & 19 & \\
\hline
\end{tabular}

Early $=$ stabilized on therapy, usually 40 weeks; late $=$ at time of reassessment, e.g. 2 years; addition of sinemet $=$ after approximately 16 weeks on sinemet.

$12 \%$ continued to show a good response and $31 \%$ a moderate response to therapy. A group of nineteen patients who had had parkinsonism for more than 10 years before L-dopa therapy showed an initial response of $47 \%$ good and $32 \%$ moderate, and after 2 years' treatment $16 \%$ continued to do well and $21 \%$ moderately well. These percentages do not permit an accurate statistical comparison but it would seem that anticholinergic and amantadine therapy has a less deleterious effect upon the ultimate response to dopamine replacement therapy than prolonged treatment with L-dopa despite the fact that many of those with a long duration of parkinsonism had the less responsive post-encephalitic form of the disease.

\section{Response to sinemet}

Twenty-four patients began treatment with sinemet: $54 \%$ were definitely improved when examined at 20 weeks and $17 \%$ moderately so. The percentage improvement to sinemet compares favourably with $49 \%$ improvement to L-dopa alone in the present series and $47 \%$ improvement obtained in an earlier study (Critchley, 1972). However, the failure rate $(29 \%)$ was also slightly higher than that encountered with L-dopa alone, though it had been anticipated that sinemet, by reducing the total dosage of $\mathrm{L}$-dopa required and lessening the gastro-intestinal an systemic side effects, would be better tolerated. The reasons for the high failure rate will be discussed later.

The advantage of sinemet was most clearly seen in the case of seventy-three patients already receiving L-dopa alone. Where sinemet had been introduced after approximately 2 years' treatment with L-dopa the response rate after 16 weeks rose from $19 \%$ to $38 \%$. The percentage improvement among the fortytwo patients receiving L-dopa for over 3 years rose from $12 \%$ to $26 \%$, and among the nineteen patients with parkinsonism of 10 years' duration and an average duration of L-dopa therapy of 2 years the replacement of L-dopa by sinemet resulted in an improvement from $16 \%$ to $26 \%$.

The response to sinemet was analysed in more 을 detail by subdividing the seventy-three patients who $\frac{7}{0}$ had previously received L-dopa alone. Twenty-three patients had shown a poor initial response to L-dopa o alone. Of these, $17 \%$ showed a favourable response $N$ to sinemet, $48 \%$ a moderate response and $35 \%$ were N unhelped. Thirty-three patients had deteriorated ${ }_{\sigma}^{\omega}$ whilst on L-dopa alone: $24 \%$ showed a good response and $39 \%$ a moderate response to sinemet. Seventeen patients had responded to L-dopa but wished to try sinemet. Three patients $(18 \%)$ did less well on sinemet but ten patients $(59 \%)$ obtained additional benefit. 


\section{Discussion}

Where L-dopa therapy is successful, the continued replenishment of striatal dopamine provides a period of stability and alleviation of symptoms which may last for about 2 years (Hunter et al., 1973). There is then a gradual erosion of the benefit obtained owing to a loss of tolerance to L-dopa. The effectiveness of L-dopa is not entirely lost and the patient may continue to function at a level superior to that before the initiation of therapy. The beneficial effects and most of the side effects experienced are dose related. The origin of many of the other unwanted effects of therapy is still a matter of conjecture, some have been attributed to an overspill of dopamine into cells bordering upon the striatum and others to a flux in the brain and blood levels of L-dopa. Such unwanted effects contribute to a variability and reduction in responsiveness which may or may not conform to a regular pattern. To obtain optimum benefit and combat the undesirable results of prolonged therapy, the physician must be prepared to manipulate the available drugs and continue to advise and encourage the patient.

The addition of carbidopa has shortened the induction time for L-dopa from approximately 16 weeks to about 10 days. Further advantages of the combined therapy are the reduction in the total dosage requirement for L-dopa and a consequent lessening of dose dependent gastro-intestinal and systemic side effects. As approximately the same concentration of L-dopa reaches the brain, the incidence of dyskinesias is not reduced. The effective response to L-dopa commences more rapidly and dramatically with the combined therapy but the onset of psychiatric disturbances, dyskinesias and 'on-off' effects involving the central nervous system is also speeded, thus patients may complain that the introduction of sinemet was responsible for the occurrence of long term and often refractory complications.

Good and excellent positive responses were obtained with sinemet even where there had been a deterioration on L-dopa. Marsden et al. (1973) claim that patients on L-dopa plus carbidopa fared better than those on L-dopa alone when observed over 1 year, but a longer term comparison has yet to be made. Many of the early failures with sinemet occurred for reasons similar to those seen with Ldopa alone, being unable to tolerate even small doses without nausea and vomiting, falling or panicking within the first week of treatment. On reflection, one wonders whether any of the other failures were due to careless management. The comparative simplicity of dosage of sinemet is deceptive. The onset of side effects is more rapid, the need for antidepressants may be overlooked, and inexperienced juniors may dismiss a patient from the clinic once an apparently satisfactory response is obtained ignoring the fact that parkinsonism is a degenerating and not a static disorder. Some of the failures with sinemet were due to the size of the prescribed preparation. The standard tablet contains the equivalent of 1000 or 1200 mg of L-dopa alone. A half tablet can be used, but there is no quarter tablet for use where it is necessary to spread and experiment with the dosage in order to overcome dyskinetic or 'on-off' effects. In these circumstances L-dopa $125 \mathrm{mg}$ or the long acting preparation Brocadopa Temtabs is more efficacious and many patients preferred to return to their previous therapy.

The aim of treatment should be to ensure that the patient is able to manage for himself until the terminal stage of the disease. This endeavour is not necessarily accomplished by using the most powerful preparation to provide the maximum benefit at any one time. The choice of preparation used, timing of medication and total dosage may be all important. For the patient with a hemi-parkinsonian tremor, stereotactic operations may still be considered. Such operations do not interfere with the consequent success of therapy but they do carry a risk of com plication which in most cases is unacceptable when compared to the safety of medical treatment. For patients with mild disability, specific treatment is not immediately indicated: amantadine may be the drug of choice and anticholinergic drugs are useful either as the sole medication or to produce a benefit in addition to that provided by L-dopa (Hughes et al., 1971; Horrocks et al., 1973). There are some indications that anticholinergic drugs may serve to prolong the period of drug tolerance and symptom alleviation.

\section{References}

BarbeaU, A. (1969) L-dopa therapy in Parkinson's Disease: a critical review of nine years' experience. Canadian Medical Association Journal, 101, 791.

Critchley, E. (1972) Levodopa and amantadine in the treatment of parkinsonism. Practitioner, 208, 499.

Horrocks, P.M., Vicary, D.J., ReEs, J.E., Parkes, J.D. \& MARSDEN, C.D. (1973) Anticholinergic withdrawal and benzhexol treatment in Parkinson's disease. Journal of Neurology, Neurosurgery and Psychiatry, 36, 936.

Hughes, R.C., Polgar, J.G., Weightman, D. \& Walton, J.N. (1971) Levodopa in parkinsonism: the effects of withdrawal of anticholinergic drugs. British Medical Journal, 2, 487.

Hunter, K.R., Laurence, D.R., Shaw, K.M. \& Stern, G.M. (1973) Sustained levodopa therapy in parkinsonism. Lancet, ii, 929.

Marden, C.D., Parkes, J.D. \& Rees, J.E. (1973) A year's comparison of treatment of patients with parkinsonism disease with levodopa combined with carbidopa versus treatment with levodopa alone. Lancet, ii, 1459. 